\title{
NOTE ON THE COMMON TANGENTS OF TWO SIMILAR CYCLOIDAL CURVES.
}

BY PROFESSOR F. MORLEY.

IN the Educational Times for November, 1895, appears a problem by Professor Aiyar,* from which it appears that from among the common tangents of two similar cycloids (not merely right cycloids, but epi- and hypocycloids) we can so select $n$ (where $n$ is the class of the curves) that three of them determine the rest; and that these $n$ common lines touch a conic. This implies that all the common tangents, - say there are $\mu n$ of them, where $\mu$ is to be found, - break up into $\mu$ sets of $n$, and determine $\mu$ conics. The fact of the common tangents separating into sets is interesting and, I think, new; and I propose to determine the configuration formed by the $\mu$ conics, using a method explained in the American Journal (vols. 15 and 16), which avoids difficulties which we should meet if we worked solely with the well-known line-equation of the curve; namely,

$$
\delta=c \cos m \omega .
$$

Working with the hypocycloid, we write its equation

$$
(p-q) x / c=p t^{-q}-q t^{p},
$$

where $p, q$ are positive integers with no common factor, and $p>q$. Here $t$ is a complex number of absolute value 1 , say a "turn"; and $x$ is therefore complex. The real constant $c$ is the radius of the vertex circle; and $t=1$ gives a vertex. The equation, in fact, maps the unit circle $|t|=1$ into the hypocycloid. The equation conjugate to $(1)$ is

$$
(p-q) y / c=p t^{q}-q t^{-p},
$$

and this and (1) are parametric equations of the curve.

The tangent at the point $t$ of the curve is

$$
x t^{q}+q t^{p}=c\left(1+t^{p+q}\right) .
$$

* Professor Ramaswami Aryar, M. A. - A variable epi- or hypocycloid $X$ similar to a given one touches three given straight lines continuously. Show that (1) the locus of the centre of $X$ is a straight line; (2) $X$ also touches $(n-3)$ other fixed lines where $n$ is the class of the curve; (3) all these $n$ lines are tangents to a conic; and (4) the envelope of the vertex-circle of $X$ is the same conic. [In particular, if a cycloid extended both ways touches three given straight lines continuously, it touches an infinite number of other lines, all of which are tangents to a parabola; the envelope of the vertex-line of the cycloid is the same parabola.] 
This is the line-equation of the curve, and is its own conjugate. To introduce homogeneous line-coördinates, we can write for any line

$$
x \xi+y \eta=\xi ;
$$

then if $\delta, \omega$ are the distance from the origin to that line, and the inclination of that distance to the line of zero-direction,

$$
\xi: \eta: \zeta=\exp -i \omega: \exp +i \omega: 2 \delta
$$

for the equation of the line is

$$
X \cos \omega+Y \sin \omega=\delta,
$$

where

$$
\begin{gathered}
X+i Y=x, \quad X-i Y=y \\
\text { i.e. } x(\cos \omega-i \sin \omega)+y(\cos \omega+i \sin \omega)=2 \delta .
\end{gathered}
$$

We have, then, for our curve

$$
\xi: \eta: \zeta=t^{q}: t^{p}: c\left(1+t^{p+q}\right),
$$

from which we can find a homogeneous line-equation if we want one.

We have supposed the curve conveniently placed, but we have to handle two such curves. We observe, then, that the most general form is obtained by writing $\tau(x-\gamma)$ for $x$, where $\tau$ is a turn and $\gamma$ is the new centre. Thus we may take two similar hypocycloids as given by the line-equations

$$
\begin{gathered}
\tau x t^{q}+y t^{p} / \tau=c\left(1+t^{p+q}\right), \\
\tau_{1}(x-\gamma) t_{1}^{q}+(y-\gamma) t_{1}^{p} / \tau_{1}=c_{1}\left(1+t_{1}^{p+q}\right),
\end{gathered}
$$

where the zero of direction is the line of centres from 0 to $\gamma, \tau$ and $\tau_{1}$ are turns which bring the curves to where they are from the positions in which vertices lie on the zero of direction, $c$ and $c_{1}$ are the vertex-radii.

To obtain common tangents, we identify these equations, and have

$$
\frac{\tau t^{q}}{\tau_{1} t_{1}^{q}}=\frac{\tau_{1} t^{p}}{\tau t_{1}^{p}}=\frac{c\left(1+t^{p+q}\right)}{c_{1}\left(1+t_{1}^{p+q}\right)+\gamma\left(\tau_{1} t_{1}^{q}+t_{1}^{p} / \tau_{1}\right)}=k,
$$

suppose, where $k$ is a turn.

Hence, eliminating $t_{1}$, we have

$$
k c_{1}\left(1+t^{p+q} / k^{2}\right)+\gamma\left(\tau t^{q}+t^{p} / \tau\right)=c\left(1+t^{p+q}\right) ;
$$

that is, $\quad\left(c-c_{1} / k\right) t^{p+q}+c-c_{1} k=\gamma\left(\tau t^{q}+t^{p} / \tau\right)$

where

$$
k^{p-q}=\left(\tau / \tau_{1}\right)^{p+q} \text {. }
$$


We have, then, $p-q$ values of $k$, and for each value $p+q$ points of the first curve, the tangents at which touch the second curve. Thus the number of common tangents of the hypocycloids is $p^{2}-q^{2}$.

But for the epicycloid we have to change the sign of $q$ throughout. We shall have, then, by the same analysis, $p+q$ values of $k$, but (5) is still of degree $p+q$; so that the number of common tangents of two similar epicycloids is $(p+q)^{2}$, the full number for curves of class $p+q$. This distinction being borne in mind, the rest of the work applies as well to the epicycloid as to the hypocycloid for which it is stated.

The equation (5), which determines a set $\Gamma_{p+q}$ of $p+q$ points, contains three constants, $c_{1}, \gamma, k$, depending on the arbitrary second curve. Therefore three of the points, and not fewer, determine the others. If the roots are not all of the form $\exp i \theta$, this merely means that some of the $p+q$ points are imaginary points of the curve.

We now find the conic which, as Professor Aiyar states, touches the tangents at $\Gamma_{p+q}$; and we shall work with the homogeneous line-coördinates defined as in (3). We have, namely,

$$
\frac{\zeta}{\tau t^{q}}=\frac{\eta}{t^{p} / \tau}=\frac{\zeta}{c\left(1+t^{p+q}\right)}=\frac{\zeta-\gamma(\xi+\eta)}{c_{1}\left(k+t^{p+q} / k\right)}
$$

or, if

$$
\zeta_{1}=\zeta-\gamma(\xi+\eta)
$$

therefore,

$$
\frac{\xi}{\tau t^{q}}=\frac{\eta}{t^{p} / \tau}=\frac{c_{1} k \zeta-c \zeta_{1}}{c c_{1}(k-1 / k) t^{p+q}}=\frac{c \zeta_{1}-c_{1} \zeta / k}{c c_{1}(k-1 / k)},
$$

$$
\begin{aligned}
c^{2} c_{1}^{2}(k-1 / k)^{2} \xi \eta & =\gamma\left(c_{1} k \zeta-c \zeta_{1}\right)\left(c \zeta_{1}-c_{1} \zeta / k\right) \\
& =c c_{1} \zeta \zeta_{1}(k+1 / k)-c_{1}{ }^{2} \zeta^{2}-c^{2} \zeta_{1}{ }^{2}
\end{aligned}
$$

In terms of $\delta, \delta_{1}$, the distances from the centre to a tangent, this is, from (3),

$$
c^{2} c_{1}^{2} \sin ^{2} \alpha=c_{1}^{2} \delta^{2}+c^{2} \delta_{1}^{2}-2 c c_{1} \delta \delta_{1} \cos \alpha
$$

where

$$
k=\exp i \alpha \text {. }
$$

Written in the form

$$
\left(c \delta_{1}-c_{1} \delta \cos \alpha\right)^{2}=c_{1}^{2} \sin ^{2} \alpha\left(c^{2}-\delta^{2}\right),
$$

the equation shows that the conic given by (7) or (8) has double contact with the vertex-circle $\delta=c$; and similarly it has double contact with the second vertex-circle $\delta_{1}=c_{1}$.

There are, of course, $p-q$ conics, which arise from the $p-q$ values of $k$ given by (6). To determine how they lie, we will first find the real foci. It is a convenient general principle that when a curve is expressed (as in (8)) by means 
114 TANGENTS OF TWO SIMILAR CYCLOIDAL CURVES. [Jan.

of the distances $\delta_{1}, \delta_{2}, \cdots$ of a tangent from fixed points $a_{1}, a_{2}, \cdots$, the real foci are the roots of the equation found by writing, in the highest terms of the line-equation, $f-a_{1}$ for $\delta_{1}, f-a_{2}$ for $\delta_{2}$, etc. Applying this to (8), we have for the real foci the quadratic

$$
c_{1}^{2} f^{2}+c^{2}(f-\gamma)^{2}-2 c c_{1} f(f-\gamma) \cos \alpha=0,
$$

where $f$ is a stroke from the centre of the first cycloid. Hence the two foci are given by

$$
\begin{aligned}
& c_{1} f / c(f-\gamma)=\cos \alpha \pm i \sin \alpha \\
&=k \text { or } 1 / k ; \\
& i . e . \text { they are } \quad f=\frac{c \gamma}{c-c_{1} k}, \bar{f}=\frac{c \gamma}{c-c_{1} / k}
\end{aligned}
$$

The inverses of the foci $f$ as to the circle $(c)$ are

$$
g=c^{2} / \bar{f}=\frac{c^{2}-c c_{1} / k}{\gamma},
$$

and therefore form a regular $(p-q)$-ad (vertices of a regular polygon) in a circle whose centre is $c^{2} / \gamma$, and radius is $c c_{1} / \gamma$. Therefore the foci $f$ themselves form a periodic $(p-q)-a d$, or "harmonic polygon," whose Hessian points or centres of inversion are the centres of the curves.

A point $g$ lies both on the directrix, corresponding to $f$, of the conic whose focus is $f$, and on the chord of contact of that conic with the vertex-circle $(c)$. For the directrix is at a distance $a / e$ from the line of centres, where $2 \alpha$ is the focal axis, $e$ the eccentricity; now from (8), when $\delta=\delta_{1}=a$, we have

and from (10),

$$
a^{2}=\frac{c^{2} c_{1}^{2} \sin ^{2} \alpha}{c^{2}+c_{1}^{2}-2 c c_{1} \cos \alpha},
$$

$$
i e \alpha=\frac{1}{2}(f-\bar{f})=\frac{i c c_{1} \gamma \sin \alpha}{c^{2}+c_{1}^{2}-2 c c_{1} \cos \alpha} ;
$$

therefore,

$$
\alpha / e=c c_{1} \sin \alpha / \gamma,
$$

and this is the ordinate of $g$.

And again, to locate the chord of contact, we have, from (9), the equation of its pole,

whence the pole is

$$
c_{1} \delta \cos \alpha=c \delta_{1},
$$

$$
x=c \gamma /\left(c-c_{1} \cos \alpha\right),
$$

and its inverse as to $(c)$ is

$$
\left(c^{2}-c c_{1} \cos \alpha\right) / \gamma
$$

which is the abscissa of $g$. 
The inverse $g_{1}$ of a focus $f$ as to the second vertex-circle $\left(c_{1}\right)$ is similarly a point on a circle with centre $c_{1}-c_{1}^{2} / \gamma$ and radius $c c_{1} / \gamma$; but observe that the angle $\alpha_{1}$ made by that radius of this circle which ends at $g_{1}$ with the line from $\gamma$ to 0 is the opposite of the angle $\alpha$ which was the corresponding angle for $g_{1}$ made by the radius from $c^{2} / \gamma$ to $g$ with the line from 0 to $\gamma$. For $\alpha$ was determined by the equation (6):

$$
(\exp i \alpha)^{p-q}=\left(\tau / \tau_{1}\right)^{p+q}
$$

$\alpha_{1}$ is determined similarly by the equation

$$
\left(\exp i \alpha_{1}\right)^{p-q}=\left(-\tau_{1} /-\tau\right)^{p+q} ;
$$

whence for each $\alpha$ there is an $\alpha_{1}$ such that

$$
\alpha+\alpha_{1}=0 .
$$

These points $g$, then, appear the proper basis for viewing the system of conics, which can be constructed as follows: We start with the two vertex-circles of radii $c, c_{1}$, and with centres $0, \gamma$. We take the inverse of each centre as to the other circle, and with these inverse points as centres, we describe equal circles of radius $c c_{1} / \gamma$. These are coaxial with the given circles (as will be shown), and are, being equal, equidistant from the radical axis $R$. In the circle whose centre is $c^{2} / \gamma$, we place any regular $(p-q)$-ad of points $g$; and we reflect this in $R$ so as to get a regular $(p-q)-a d$ of points $g_{1}$ in the other equal circle. Taking any pair of points $g, g_{1}$, let the lines $\overline{0 g}$, $\overline{\gamma g}$ meet at $f$. The conic whose focus is $f$, whose directrix is $g g_{1}$, and whose centre is on the line of centres $\overline{0 \gamma}$, is one of the system. The $p-q$ pairs of points $g, g_{1}$ give by the same construction the $p-q$ conics.

It remains to prove the elementary fact that the equal circles are coaxial with the given ones $(c)$ and $\left(c_{1}\right)$. The foci of all conics which are bitangent with two circles, when they are conjugate as to the line of centres, lie on the circle of similitude of $(c)$ and $\left(c_{1}\right)$. This was remarked by Roberts (Examples on Conics, p. 86), and, in fact, appears from (10), for from (10)

$$
|f /(f-\gamma)|=\left|c / c_{1}\right| ;
$$

that is, the circles $(c)$ and $\left(c_{1}\right)$ appear of the same size from all points $f$. Now we know that the circle of similitude is coaxial with $(c)$ and $\left(c_{1}\right)$; that is, it passes through their real or imaginary intersections. Naturally, then, its inverse as to either circle $(c)$ or $\left(c_{1}\right)$ will pass through the same intersections. That is, the fact is evident for real intersections; but an algebraic proof would apply as much to imaginary as to real intersections. 
The conic (7) depends solely on $k$; that is, on $\tau / \tau_{1}$; that is, on the relative orientation of the two curves; but $\tau$, and therefore the actual orientation of the first curve, disappeared in finding the conic. Hence if we now let the two curves rotate about their centres with equal angular velocities, the envelope of their common tangents is the entire system of conics.

Haverford, Pa.

\section{NOTES.}

The Annual Meeting of the American Mathematical Socresy was held in New York on Friday afternoon, December 27, at three o'clock, the President, Dr. HILL, in the chair. There were twenty-five members present. On the recommendation of the Council, the following persons, nominated at the preceding meeting, were elected to membership: Mr. Julius L. Neuferd, Central High School, Philadelphia, Pa.; Mr. Wendell Melville Strong, Yale University, New Haven, Conn. Reports were presented by the Secretary and Treasurer. The Secretary stated that the membership of the Society was 267 . The average attendance at the ordinary meetings during the year had been 15 , the attendance at the last annual meeting 17 , and that at the summer meeting 26 . The number of members who had attended at least one meeting during the year was 61. Professor Cole, Dr. Chittenden, and Professor Ladue were appointed an auditing committee to examine the Treasurer's accounts.

The chair appointed Mr. Legras and Mr. Lambert tellers for the annual election. Upon examining the ballots cast in person and by mail, they announced that the following ticket had been elected:

President: Dr. George W. HiLl.

Vice-President: Professor Hubert A. Newton.

Secretary: Professor Frank N. Cole.

Treasurer: Professor R. S. WoodwaRd.

Librarian: $\quad$ Professor Pomeroy Ladue.

Committee of Publication:

Professor Thomas S. Fiske.

Professor Alexander Ziwet.

Professor Frank Morley.

Three Members of the Council to serve until December, 1898 :

Professor E. W. Hyde.

Professor W. Woolsey Johnson.

Professor B. O. Peirce. 